\title{
ATIVIDADE INSETICIDA DE Cunila angustifolia SOBRE ADULTOS DE Acanthoscelides obtectus EM LABORATÓRIO
}

\section{INSECTICIDAL ACTIVITY OF Cunila angustifolia ON Acanthoscelides obtectus ADULTS UNDER LABORATORY CONDITIONS}

\author{
${ }^{\bullet}$ Marcoandre Savaris ${ }^{1}$, Silvana Lampert ${ }^{1}$, Flávio Roberto Mello García ${ }^{2}$, Sandra Mara Sabedot-Bordin ${ }^{3}$ e \\ Neuza Fernandes de Moura ${ }^{3}$ \\ ${ }^{1}$ Universidade de Passo Fundo, Programa de Pós-graduação em Agronomia, Laboratório de Entomologia, \\ Caixa postal 611, 99001-970, Passo Fundo, Rio Grande do Sul, Brasil. • masavaris@yahoo.com.br \\ ${ }^{2}$ Universidade Federal de Pelotas, Instituto de Biologia. Departamento de Zoologia e Genética, Laboratório \\ de Ecologia de Insetos. Caixa postal 35, CEP 96010-900, Pelotas, Rio Grande do Sul. \\ ${ }^{3}$ Universidade Comunitária da Região de Chapecó, Programa de Pós-graduação em Ciências Ambientais, \\ Laboratório de Entomologia. Caixa postal 747, CEP 89809000, Chapecó, Santa Catarina.
}

\section{Resumo}

A utilização de extratos e óleos essenciais de plantas tem-se constituído em uma importante alternativa para controle de insetos-praga de grãos armazenados. O objetivo deste trabalho foi avaliar o efeito inseticida de extratos e do óleo essencial de $C$. angustifolia sobre adultos de $A$. obtectus em condições de laboratório. $\mathrm{O}$ experimento foi conduzido em delineamento inteiramente casualizado com quatro repetições, onde foram testados os extratos bruto e aquoso, fração hexânica e óleo essencial de $C$. angustifolia nas doses $0.001,0.002,0.004$ e $0.008 \mathrm{~mL} \mathrm{~cm}^{-2}$. Cada repetição foi constituída de 10 adultos de $A$. obtectus. A mortalidade dos insetos foi registrada as 24, 48, 72, 96 e 120 horas após a aplicação. Todas as doses do óleo essencial de C. angustifolia apresentaram eficiência de $100 \%$ na mortalidade de A. obtectus após 24 horas. Os demais tratamentos apresentaram baixa eficiência, quando comparado ao efeito do óleo essencial. Este estudo evidencia a ação inseticida do óleo essencial de $C$. angustifolia demonstrando ser promissor no controle desta praga.

Palavras chave: Ação inseticida, inseto-praga, armazenamento, planta inseticida, caruncho-do-feijão.

Abstract

$\mathrm{T}$ he use of extracts and essential oils of plants has been used as an important alternative to control insect pests of stored grains. The aim of this study was to evaluate the insecticide effect of extracts and the essential oil of C. angustifolia on adults of $A$. obtectus, under laboratory conditions. The experiment was conducted at random design with four replications, being tested crude and aqueous extracts, hexanic fraction and essential oil of C. angustifolia, at the doses of 0.001, 0.002, 0.004 and $0.008 \mathrm{~mL} \mathrm{~cm}^{-2}$. Each replicate received 10 adults of $A$. obtectus. Mortality of these insects was recorded the 24, 48, 72, 96 and 120 hours after application. All doses of the essential oil of C. angustifolia achieved $100 \%$ of efficiency in the mortality of $A$. obtectus after 24 hours. The other treatments showed low control efficiency when they were compared to the effect of essential oil. This research demonstrates the insecticide activity of the essential oil of $C$. angustifolia proving to be promising in the control of this primary pest.

Key words: Insecticide action, insect pest, storage, insecticide plant, bean weevil.

\section{INTRODUÇ̃̃o}

A utilização de plantas inseticidas é atualmente um dos métodos alternativos mais estudados para controle de insetos em produtos armazenados (Procópio et al., 2003). As substâncias de origem vegetal apresentam diversas vantagens quando comparadas aos inseticidas sintéticos; reduzem a persistência e a acumulação de

Recibido: 22-Diciembre-2011. Recibido en forma corregida: 15-Febrero-2012. Aceptado: 28-Febrero-2012.

Publicado como ARTÍCULO CIENTÍFICO en Ciencia y Tecnología 5(1): 1-5. 2012 resíduos no ambiente, tem maior seletividade, são biodegradáveis e não apresentam efeitos colaterais típicos dos inseticidas convencionais (Gionetto e Chávez, 2000).

Dentre os vegetais conhecidos com elevado potencial químico, encontra-se a família Lamiaceae 
com aproximadamente 264 gêneros descritos (USDA, 2011). Dentre eles o gênero Cunila L. conhecido por apresentar óleos voláteis, e constituintes químicos como o linalol, cineol, mentofurana, limonero, isomentonas, mentona e pulegona (Manns, 1995; Bordignon et al., 1997; Echeverrigaray et al., 2003).

Cunila angustifolia Bentham, 1834 é uma espécie nativa da América Tropical, com ampla distribuição por todo o mundo (Xifreda e Mallo, 2001). Apresenta como principais metabólitos secundários sabineno, $\gamma$-terpineno e limoneno (Echeverrigaray et al., 2003). Bordignon et al. (1999), determinaram como constituintes majoritários desta espécie o sabineno (41.4\%) e o óxido-de-transpiperitona, (42.4\%), já para Moreira e Krambeck (1976) e Prado (2007), o composto majoritário foi a pulegona, demonstrando que há uma relação significativa entre a composição química e a origem geográfica da espécie $C$. angustifolia. Compostos orgânicos bioativos produzidos por vegetais incluem repelentes, inibidores de crescimento, esterilizantes e toxinas, que formam uma vasta defesa química contra insetos e patógenos invasores (Cavalcante et al., 2006).

Este trabalho tem por objetivo avaliar o efeito inseticida de extratos e do óleo essencial de $C$. angustifolia sobre adultos de $A$. obtectus em condições de laboratório.

\section{Material e MÉtodos}

\section{Coleta do material}

$\mathrm{R}$ amos e folhas de C. angustifolia (Figura 1A) foram coletados em propriedades rurais no município de Chapecó, Santa Catarina (-2708'50.86”S $-52^{\circ} 38^{\prime} 17.54$ 'W). O material botânico foi identificado pela doutora Thais Scotti do Canto-Dorow e a exsicata depositada sob número SMDB 9925 no herbário da Universidade Federal de Santa Maria (UFSM). Adultos

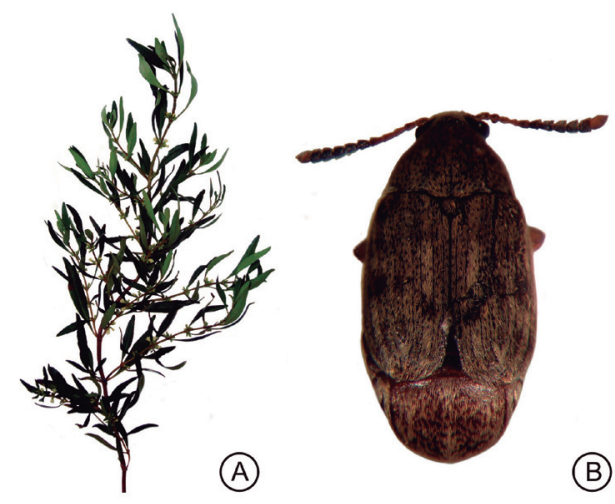

Figura 1. Ramo de Cunila angustifolia (A), Vista dorsal de Acanthoscelides obtectus (B). de A. obtectus (Figura 1B) foram coletados em grãos armazenados de feijão Phaseolus vulgaris L. no mesmo município, acondicionados no Laboratório de Entomologia da Universidade Comunitária Regional de Chapecó (UNOCHAPECÓ), e a identificação realizada com base na chave de Pereira e Salvadori (2006).

\section{Obtenção e composição do óleo e extratos}

O óleo volátil das folhas foi obtido através de destilação por arraste de vapor por quatro horas, utilizando um aparelho de "Clevenger" modificado, tendo um rendimento de $0.9 \%$ de óleo. Posteriormente foi submetido à análise por Cromatografia Gasosa, equipado com coluna de sílica fundida $(25 \mathrm{~m} \times 0.25$ $\mathrm{mm}, 0.25 \mu \mathrm{m})$ recheada com SE-54. As condições para

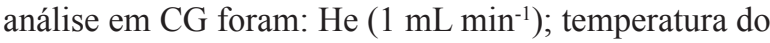
injetor $200^{\circ} \mathrm{C}$; temperatura FID $280^{\circ} \mathrm{C}$; temperatura da coluna $50-250^{\circ} \mathrm{C} \mathrm{a} 4^{\circ} \mathrm{C} \mathrm{min}^{-1}$. As análises em CG/EM foram realizadas utilizando o modelo HP 5973-6890 GC-MSD operando em modo E.I a 70 e V, equipado com coluna capilar HP-5 (30 m x $0.25 \mathrm{~mm}, 0.25 \mu \mathrm{m})$.

Para a obtenção dos extratos, folhas de $C$. angustifolia foram secas a sombra, maceradas e extraídas por percolação com etanol água ${ }^{-1}$ exaustivamente. O solvente foi evaporado a pressão reduzida, em evaporador rotativo Ramdolph, resultando em um resíduo escuro e viscoso, sendo denominado de extrato bruto. Para a obtenção do extrato aquoso foi realizado o mesmo procedimento, porém a extração foi realizada apenas com água. $\mathrm{O}$ extrato bruto foi dissolvido em uma solução hidro-alcóolica, sofrendo partição com hexano, e extraído exaustivamente até obtenção da fração hexânica. Foram realizadas as diluições do óleo e extratos na proporção de $1 \mathrm{~g}$ da solução para $4 \mathrm{~mL}$ do solvente. Para extração do óleo foi utilizado éter etílico $\left(\mathrm{C}_{2} \mathrm{H}_{5}\right)_{2}$ como solvente, enquanto o extrato bruto e a fração hexânica foram extraídas com álcool metílico $\left(\mathrm{CH}_{3} \mathrm{OH}\right)$ e o extrato aquoso em água destilada. A identificação dos compostos foi realizada, com base na literatura, comparando-se o espectro de massa e o índice de retenção, calculado por enriquecimento com uma mistura de hidrocarbonetos.

\section{Aplicação do óleo e extratos em laboratório}

O experimento foi conduzido, sob temperatura de $25 \pm 2^{\circ} \mathrm{C}$ e umidade relativa de $70 \pm 10 \%$ com fotofase de 12 horas. Com auxílio de pipetas as soluções foram separadas em doses de 0.001, 0.002, 0.004 e 0.008 $\mathrm{mL} \mathrm{cm}{ }^{-2}$, e em seguida impregnadas em papel de filtro de $7 \mathrm{~cm}$ de diâmetro, que foram colocados no interior de placas de Petry, conforme o método descrito por 
Takematsu (1983). Cada repetição foi composta de 10 adultos de $A$. obtectus, que foram avaliados às $24,48,72$, 96 e 120 horas após a aplicação. O ensaio foi realizado sob um delineamento inteiramente casualizado em arranjo fatorial (4 x 4 x 5) com quatro repetições. Um dos fatores foram os extratos, com quatro níveis (extrato aquoso, bruto, fração hexânica e óleo essencial) em quatro subníveis (doses 0.001, 0.002, 0.004 e 0.008 $\mathrm{mL} \mathrm{cm} \mathrm{cm}^{-2}$ ), o outro fator, o tempo de exposição aos insetos, com cinco níveis (24, 48, 72, 96 e 120 horas). A análise estatística foi realizada utilizando-se o software Assistat (Silva e Azevedo, 2009). Foi realizado análise da variância avaliando-se o número de insetos vivos. Os valores obtidos foram transformados em raiz quadrada de $\mathrm{X}+0.5$, e a normalidade dos dados verificada pelo teste de Shapiro-Wilk. As médias obtidas foram comparadas pelo teste de Tukey, ao nível de 5\% de probabilidade de erro. A eficiência dos diferentes tratamentos foi obtida pela equação de Abbott (1925).

\section{Resultados E discussão}

\section{Caracterização dos constituintes voláteis de Cunila angustifolia}

$\mathrm{A}^{\mathrm{a}}$ análise dos constituintes voláteis da $C$. angustifolia permitiu identificar $93.4 \%$ dos constituintes presentes na espécie. De acordo com esta análise a substância majoritária foi a pulegona $(56.1 \%)$, seguida de isomentona $(11.2 \%)$, $\beta$-cariofileno $(6.8 \%)$, neomentol $(5.7 \%)$ e do mentol $(3.6 \%)$.

\section{Bioensaio com Acanthoscelides obtectus}

Todas as doses do óleo essencial de C. angustifolia apresentaram eficiência de $100 \%$ na mortalidade de adultos de $A$. obtectus, 24 horas após a exposição aos insetos (Tabela 1). Nos tratamentos com extratos e a fração hexânica a eficiência foi menor que $16 \%$. A atividade inseticida do óleo essencial é atribuída à presença do monoterpeno pulegona, constituinte majoritário do óleo essencial utilizado nas avaliações. Resultado que corrobora o encontrado neste estudo foi obtido por Prado (2007), que obteve a pulegona como constituinte majoritário do óleo essencial de C. angustifolia, relatando alta toxicidade frente à Alphitobius diaperinus, apresentando nas concentrações de $5 \%$ e $10 \%$ eficiência de $100 \%$ nas primeiras 24 horas. Lee et al. (2003) estudando o efeito fumegante do monoterpeno pulegona, comentam o efeito tóxico sobre adultos de Sitophilus oryzae, Tribolium castaneum, Oryzaephilus surinamensis, Musca domestica e Blattella germanica, observando $100 \%$ de mortalidade para as cinco espécies.

De modo geral, os extratos e a fração hexânica de $C$. angustifolia, nas doses avaliadas, apresentaram baixa eficiência de controle sobre adultos de A. obtectus, quando comparado ao efeito do óleo essencial (Tabela 1). A mortalidade máxima observada foi de $69 \%$ dos insetos expostos à fração hexânica, na dose de 0.008 $\mathrm{mL} \mathrm{cm} \mathrm{cm}^{-2}$, após 120 horas de exposição dos insetos aos diferentes tratamentos. Os resultados obtidos são inferiores aos encontrados por Smaniotto et al. (2010), para adultos de $A$. obtectus, que citam eficiência de

Tabela 1. Número médio de adultos vivos de Acanthoscelides obtectus após a aplicação de extrato aquoso, bruto, fração hexânica e óleo essencial de Cunila angustifolia em laboratório $\left(\mathrm{T}=25 \pm 2^{\circ} \mathrm{C}\right.$, UR $=70 \pm 10 \%$ e fotofase $12 \mathrm{~h}) . \mathrm{EC}=$ eficiência de controle

\begin{tabular}{|c|c|c|c|c|c|c|c|c|c|c|c|}
\hline \multirow{2}{*}{$\begin{array}{l}\text { Tratamen- } \\
\text { tos }\end{array}$} & \multirow{2}{*}{$\begin{array}{c}\text { Dose } \\
\left(\mathrm{mL} \mathrm{cm}^{-2}\right)\end{array}$} & \multicolumn{10}{|c|}{ Horas após a aplicação } \\
\hline & & 24 & $\begin{array}{l}\mathrm{EC} \\
(\%) \\
\end{array}$ & 48 & $\begin{array}{l}\mathrm{EC} \\
(\%) \\
\end{array}$ & 72 & $\begin{array}{l}\mathrm{EC} \\
(\%) \\
\end{array}$ & 96 & $\begin{array}{l}\mathrm{EC} \\
(\%) \\
\end{array}$ & 120 & $\begin{array}{l}\mathrm{EC} \\
(\%) \\
\end{array}$ \\
\hline \multirow{4}{*}{$\begin{array}{l}\text { Extrato } \\
\text { aquoso }\end{array}$} & 0.001 & $10.0 \pm 0.0 \mathrm{~b}$ & 0 & $9.2 \pm 0.2 \mathrm{~cd}$ & 8 & $6.6 \pm 0.2 \mathrm{bcd}$ & 34 & $5.6 \pm 0.3$ & 44 & $5.4 \pm 0.3 \quad \mathrm{~cd}$ & 46 \\
\hline & 0.002 & $9.70 \pm 0.2 b$ & 3 & $8.7 \pm 0.2 \mathrm{~cd}$ & 13 & $7.7 \pm 0.3 \mathrm{~cd}$ & 23 & $6.4 \pm 0.2$ & 36 & $4.6 \pm 0.2 \mathrm{bcd}$ & 54 \\
\hline & 0.004 & $9.20 \pm 0.3 b$ & 8 & $7.9 \pm 0.4 \mathrm{~cd}$ & 21 & $6.0 \pm 0.1 \mathrm{bc}$ & 40 & $4.7 \pm 0.2 \mathrm{~cd}$ & 53 & $3.6 \pm 0.2 \mathrm{bc}$ & 64 \\
\hline & 0.008 & $8.90 \pm 0.2 \mathrm{~b}$ & 11 & $7.7 \pm 0.3 \mathrm{~cd}$ & 23 & $5.3 \pm 0.1 \mathrm{bc}$ & 47 & $3.6 \pm 0.1 \mathrm{~b}$ & 64 & $3.4 \pm 0.1 \mathrm{bc}$ & 66 \\
\hline \multirow{4}{*}{$\begin{array}{l}\text { Extrato } \\
\text { bruto }\end{array}$} & 0.001 & $9.40 \pm 0.3 b$ & 6 & $9.4 \pm 0.4 \quad d$ & 6 & $7.4 \pm 0.2 \mathrm{~cd}$ & 26 & $6.3 \pm 0.2$ & 37 & $5.8 \pm 0.3$ & 42 \\
\hline & 0.002 & $8.90 \pm 0.2 b$ & 11 & $8.4 \pm 0.3 \mathrm{~cd}$ & 16 & $7.7 \pm 0.3 \mathrm{~cd}$ & 23 & $5.9 \pm 0.3$ & 41 & $5.2 \pm 0.2 \mathrm{~cd}$ & 48 \\
\hline & 0.004 & $10.0 \pm 0.0 \mathrm{~b}$ & 0 & $9.2 \pm 0.2 \mathrm{~cd}$ & 8 & $7.4 \pm 0.2 \mathrm{~cd}$ & 26 & $6.1 \pm 0.3$ & 39 & $4.0 \pm 0.2 \mathrm{bcd}$ & 60 \\
\hline & 0.008 & $9.20 \pm 0.2 \mathrm{~b}$ & 8 & $8.2 \pm 0.3 \mathrm{~cd}$ & 18 & $6.4 \pm 0.2 \mathrm{bcd}$ & 36 & $4.8 \pm 0.2 \mathrm{~cd}$ & 52 & $4.0 \pm 0.2 \mathrm{bcd}$ & 60 \\
\hline
\end{tabular}

continúa en la siguiente página 
Savaris et al.

\begin{tabular}{|c|c|c|c|c|c|c|c|c|c|c|c|}
\hline \multirow{2}{*}{$\begin{array}{l}\text { Tratamen- } \\
\text { tos }\end{array}$} & \multirow{2}{*}{$\begin{array}{c}\text { Dose } \\
\left(\mathrm{mL} \mathrm{cm}^{-2}\right)\end{array}$} & \multicolumn{10}{|c|}{ Horas após a aplicação } \\
\hline & & 24 & $\begin{array}{l}\text { EC } \\
(\%)\end{array}$ & 48 & $\begin{array}{l}\text { EC } \\
(\%)\end{array}$ & 72 & $\begin{array}{l}\text { EC } \\
(\%)\end{array}$ & 96 & $\begin{array}{l}\text { EC } \\
(\%)\end{array}$ & 120 & $\begin{array}{l}\text { EC } \\
(\%)\end{array}$ \\
\hline \multirow{4}{*}{$\begin{array}{l}\text { Fração } \\
\text { hexânica }\end{array}$} & 0.001 & $9.70 \pm 0.2 b$ & 3 & $9.2 \pm 0.2 \mathrm{~cd}$ & 8 & $8.7 \pm 0.3 \quad \mathrm{~d}$ & 13 & $6.4 \pm 0.2$ & 36 & $6.0 \pm 0.3$ & 40 \\
\hline & 0.002 & $9.70 \pm 0.2 b$ & 3 & $8.7 \pm 0.2 \mathrm{~cd}$ & 13 & $6.6 \pm 0.2 \mathrm{bcd}$ & 34 & $4.6 \pm 0.2 \mathrm{bc}$ & 54 & $4.4 \pm 0.1 \mathrm{bcd}$ & 56 \\
\hline & 0.004 & $9.20 \pm 0.3 b$ & 8 & $8.7 \pm 0.2 \mathrm{~cd}$ & 13 & $6.1 \pm 0.1 \mathrm{bc}$ & 39 & $4.6 \pm 0.2 \mathrm{bc}$ & 54 & $3.7 \pm 0.2 \mathrm{bc}$ & 63 \\
\hline & 0.008 & $8.40 \pm 0.1 \mathrm{~b}$ & 16 & $6.6 \pm 0.1 \mathrm{~b}$ & 34 & $4.7 \pm 0.1 \mathrm{~b}$ & 53 & $3.4 \pm 0.1 \mathrm{~b}$ & 66 & $3.1 \pm 0.1 \mathrm{~b}$ & 69 \\
\hline \multirow{4}{*}{$\begin{array}{c}\text { Óleo } \\
\text { essencial }\end{array}$} & 0.001 & $0.00 \pm 0.0 \mathrm{a}$ & 100 & $0.0 \pm 0.0 \mathrm{a}$ & 100 & $0.0 \pm 0.0 \mathrm{a}$ & 100 & $0.0 \pm 0.0 \mathrm{a}$ & 100 & $0.0 \pm 0.0 \mathrm{a}$ & 100 \\
\hline & 0.002 & $0.00 \pm 0.0 \mathrm{a}$ & 100 & $0.0 \pm 0.0 \mathrm{a}$ & 100 & $0.0 \pm 0.0 \mathrm{a}$ & 100 & $0.0 \pm 0.0 \mathrm{a}$ & 100 & $0.0 \pm 0.0 \mathrm{a}$ & 100 \\
\hline & 0.004 & $0.00 \pm 0.0 \mathrm{a}$ & 100 & $0.0 \pm 0.0 \mathrm{a}$ & 100 & $0.0 \pm 0.0 \mathrm{a}$ & 100 & $0.0 \pm 0.0 \mathrm{a}$ & 100 & $0.0 \pm 0.0 \mathrm{a}$ & 100 \\
\hline & 0.008 & $0.00 \pm 0.0 \mathrm{a}$ & 100 & $0.0 \pm 0.0 \mathrm{a}$ & 100 & $0.0 \pm 0.0 \mathrm{a}$ & 100 & $0.0 \pm 0.0 \mathrm{a}$ & 100 & $0.0 \pm 0.0 \mathrm{a}$ & 100 \\
\hline
\end{tabular}

Médias seguidas pela mesma letra não apresentam diferenças estatísticas (Tukey, $\mathrm{p}<0.05$ ). $\mathrm{CV}=11.2$

$100 \%$ na mortalidade dos insetos submetidos ao extrato bruto de folhas de Cabralea canjerana. Também diferem dos obtidos por Procópio e Vendramim (1997) que obtiveram resultados promissores ao observarem mortalidade de $100 \%$ em adultos de A. obtectus, ocasionado por pós de folhas, flores e frutos (em conjunto) de Chenopodium ambrosioides.

\section{ConClusões}

A análise dos constituintes voláteis da $C$. angustifolia revelou como constituinte majoritário a pulegona (56.1\%). O monoterpeno pulegona através do óleo essencial apresentou efeito inseticida sobre adultos de $A$. obtectus, sendo a sua utilização no controle desta praga considerada promissora. Entretanto, deve-se estudar melhor qual a dose ideal, já que o efeito das doses 0.001 e $0.008 \mathrm{~mL} \mathrm{~cm}^{-2}$ sobre os insetos foi similar. Os extratos e a fração hexânica de $C$. angustifolia, em todas as doses avaliadas apresentaram baixa eficiência de controle, quando comparado ao efeito do óleo essencial.

\section{LiTERATURA CITADA}

Abbott, W. S. 1925. A method of computing the effectiveness of an insecticide. Journal of economic entomology. 18(2):265-267.

Bordignon, S. A. L., E. P. Schenkel \& V. Spitzer. 1997. The essential oil composition of Cunila microcephala and Cunila fasciculata. Phytochemistry. 44(7):1283-1286.

Bordignon, S. A. L., E. P. Schenkel \& V. Spitzer. 1999. The essential oil composition of Cunila angustifolia (Lamiaceae). Journal of Essential Oil Research. 11:145-148.

Cavalcante, G. M., A. F. C. Moreira \& S. D. Vasconcelos. 2006. Potencialidade inseticida de extratos aquosos de essências florestais sobre mosca- branca. Pesquisa Agropecuária Brasileira. 41:914.

Echeverrigaray, S., F. Fracaro, A. C. A. Santos, N. Paroul, R. Wasum \& L. A. Serafini. 2003. Essential oil composition of south Brazilian populations of Cunila galioides and its relation with the geographic distribution. Biochemical systematics and ecology. 31:467-475.

Gionetto, F. \& E. C. Chávez. 2000. Desarrollo actual de lãs investigaciones alelopaticas de la producción de inseticidas botánicos en Michoacán (México). In: Simposio nacional sobre substancias vegetales y minerales en el combate de plagas, 6., 2000, Acapulco. Memórias Acapulco: SME, 2000. p. 123-134

Lee, S., C. J. Peterson, R. R. Coats. 2003. Fumigation toxicity of monoterpenoids to several stored product insects. Journal of Stored Products Research. 39:77-85.

Manns, D. 1995. Linalool and cineole type glucosides from Cunila spicata. Phytochemistry. 39(5):11151118.

Moreira, E. A. \& R. Krambeck. 1976. Análise cromatográfica de óleo essencial das folhas de Cunila angustifolia Benth. Labiatae. Tribuna Farmacêutica. 44:50-59.

Pereira, P. R. V. S. \& J. R. Salvadori. 2006. Identificação dos principais coleóptera (Insecta) associados a produtos armazenados. Passo Fundo: Embrapa Trigo. 33 p. (Embrapa Trigo. Documentos Online, 75). Disponível em: http://www.cnpt.embrapa.br/ biblio/do/p_do75.htm.

Prado, G. P. do. 2007. Caracterização química e bioatividade do óleo essencial de Cunila angustifolia Benth (Lamiaceae) sobre Alphitobius diaperinus (Panzer, 1797) (Coleoptera: Tenebrionidae). Chapecó. 60p. Dissertação (Mestrado em Ciências Ambientais) 
- Universidade Comunitária Regional de Chapecó - UNOCHAPECÓ.

Procópio, S. O. \& J. D. Vendramim. 1997. Avaliação da atividade inseticida de diversos pós de origem vegetal em relação à Acanthoscelides obtectus (Say.) In: Congresso Brasileiro de Entomologia, 16., Salvador, 1997. Resumos. Salvador: SBE. p 326.

Procópio, S. O., J. D. Vendramim, J. I. Ribeiro Júnior \& J. B. Santos. 2003. Bioatividade de diversos pós de origem vegetal em relação à Sitophilus zeamais Mots. (Coleoptera: Curculionidae). Ciência e Agrotecnologia. 27(6):1231-1236.

Silva, F. A. S. \& C. A. V. de Azevedo. 2009. Principal components analysis in the software AssistatStatistical Attendance. In: World congress on computers in agriculture conference, 7, Reno, NV, USA: American Society of Agricultural and Biological Engineers.

Smaniotto, L., N. F. de Moura, R. B. N. Denardin \& F. R. M. Garcia. 2010. Bioatividade de Cabralea canjerana (Vell.) Mart. (Meliaceae) no controle de adultos de Acanthoscelides obtectus (Coleoptera, Bruchidae) em laboratório. Biotemas. 23:31-35.
Takematsu, A. P. 1983. Suscetilidade de Sitophilus zeamais Mots, 1855 (Coleoptera-Curculionidae) de diferentes regiões do Estado de São Paulo, a inseticidas fosforados e piretróides em condições de laboratório. Piracicaba. 77p. Dissertação (Mestrado) - Escola Superior de Agricultura "Luiz de Queiroz", Universidade de São Paulo.

USDA, ARS. 2011. National Genetic Resources Program. Germplasm Resources Information Network - (GRIN) [Online Database]. National Germplasm Resources Laboratory, Beltsville, Maryland. URL: http://www.ars-grin.gov/cgibin/npgs/html/family.pl?2099.

Xifreda, C. C. \& A. C. Mallo. 2001. Citas nuevas o críticas para la flora argentina III: Cunila angustifolia (Lamiaceae: Mentheae). Darwiniana. 39(1-2):175-178. 